\title{
Development of an ex vivo model of endovenous laser ablation of the great saphenous vein in a pilot study ${ }^{1}$
}

\author{
Walter Junior Boim Araujo, Jorge Rufino Ribas Timi' ${ }^{\mathrm{II}}$, Fabiano Luiz Erzinger ${ }^{\mathrm{III}}$, Filipe Carlos Caron ${ }^{\mathrm{IV}}$, Alan Knolseisen \\ Cambrussi $^{\mathrm{V}}$
}

DOI: http://dx.doi.org/10.1590/S0102-865020160030000003

${ }^{I}$ Fellow PhD degree, Postgraduate Program in Clinical Surgery, Department of Surgery, Universidade Federal do Paraná (UFPR), Curitiba-PR, Brazil. Scientific, intellectual, conception and design of the study; acquisition and interpretation of data; technical procedures; histopathological examinations; statistics analysis; manuscript preparation.

IIPhD, Associate Professor, Vascular Surgery, Department of Surgery, UFPR, Curitiba-PR, Brazil. Scientific, intellectual, conception and design of the study, critical revision, final approval.

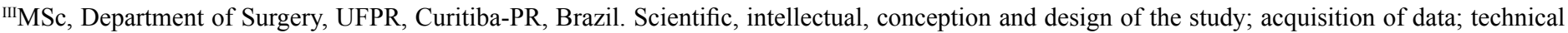
procedures; manuscript writing; final approval.

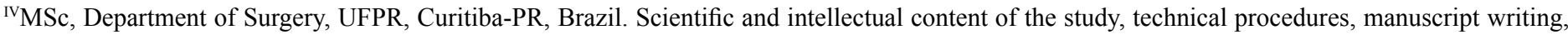
final approval.

${ }^{v}$ MD, Vascular Surgeon, Hospital Angelina Caron, Campina Grande do Sul-PR, Brazil. Scientific and intellectual content of the study, technical procedures, manuscript writing, final approval.

\begin{abstract}
PURPOSE: To develop an ex vivo model for the analysis of macroscopic, histological and immunohistochemical changes after experimental endovenous laser ablation (EVLA) of the great saphenous vein (GSV).

METHODS: We describe a model produced with glass tubes and introducer sheaths to mimic the physiological conditions of EVLA procedures, such as tumescence and blood flow. A pilot study was conducted to evaluate an ex vivo procedure of EVLA of an incompetent GSV segment using a 1470-nm radial fiber diode laser (7 W power) and an automatic pull-back device. The vein segment was analyzed macroscopically and by hematoxylin \& eosin staining, elastic fiber histochemistry, Gomori's trichrome staining, and alpha-smooth muscle actin immunohistochemistry.
\end{abstract}

RESULTS: No perforations were observed macroscopically. No muscle cell adhesion was observed in the central part of the ablated vein, showing tissue disruption. There was low labeling for elastic fibers, disruption of muscle fibers, and a reduced expression of the specific marker for this cell type.

CONCLUSION: This ex vivo endovenous laser ablation model is a low cost alternative to in vivo experiments, providing standardized experimental conditions.

Key words: Varicose Veins. Laser Therapy. Ablation Techniques. 


\section{Introduction}

Chronic venous insufficiency caused by varicose veins is a common medical condition, with prevalence rates ranging as high as $28 \%$ to $35 \%$ in adults ${ }^{1}$. Morbidity and patient dissatisfaction associated with conventional surgical treatment of great saphenous vein (GSV) reflux, which includes high ligation at the saphenofemoral junction (SFJ) combined with stripping of the GSV, have led to the development of alternative techniques ${ }^{2}$.

Currently, endovenous treatment is preferred to conventional surgical treatment, and several successful treatment options are available for patients with chronic venous insufficiency caused by varicose veins. Because of the various options available, many physicians have had difficulties in choosing the best method that meets their patients' needs. In addition, the inter-method variability of protocols and parameters used in endovenous treatment should also be considered, as these factors might hinder effective decision-making.

If on the one hand this variability may lead to different opinions about which method of endovenous treatment is the best choice, on the other hand it should prompt research on and development of standards for the use of these methods. Innovative concepts and alternative treatments should therefore be developed, tested and evaluated until their approval for clinical use. In this context, experimental models are useful tools to investigate the mechanisms involved in endovenous laser ablation (EVLA) procedures because they can mimic basic processes that occur in GSV reflux and provide an alternative to studies in humans and in animal models.

Experimental models may also be used for training purposes and scientific research, allowing the analysis of acute effects on ablated veins based on macroscopic, histological and immunohistochemical findings. In addition, these models do not require the same approval process as research involving animals or human subjects, thus minimizing ethical concerns and reducing the costs for implementation.

In order to investigate the mechanisms involved in EVLA and to test treatment strategies, the aim of the current study was to develop a simple ex vivo model of EVLA of the GSV that mimics the physiological conditions of the procedure, such as tumescence and blood flow, and to evaluate macroscopic, histological and immunohistochemical changes in the ablated GSV segment.

\section{Methods}

This was a descriptive study using an experimental design. The study was approved on June 1, 2015 by the Research Ethics Committee of Hospital Angelina Caron, a teaching hospital affiliated with Universidade Federal do Paraná (project approval No. 45209015.9.0000.5226). All experiments were conducted in accordance with the National Institutes of Health guidelines.

\section{Ex vivo model of EVLA of the GSV}

The materials used to produce the experimental model are shown in Figure 1.

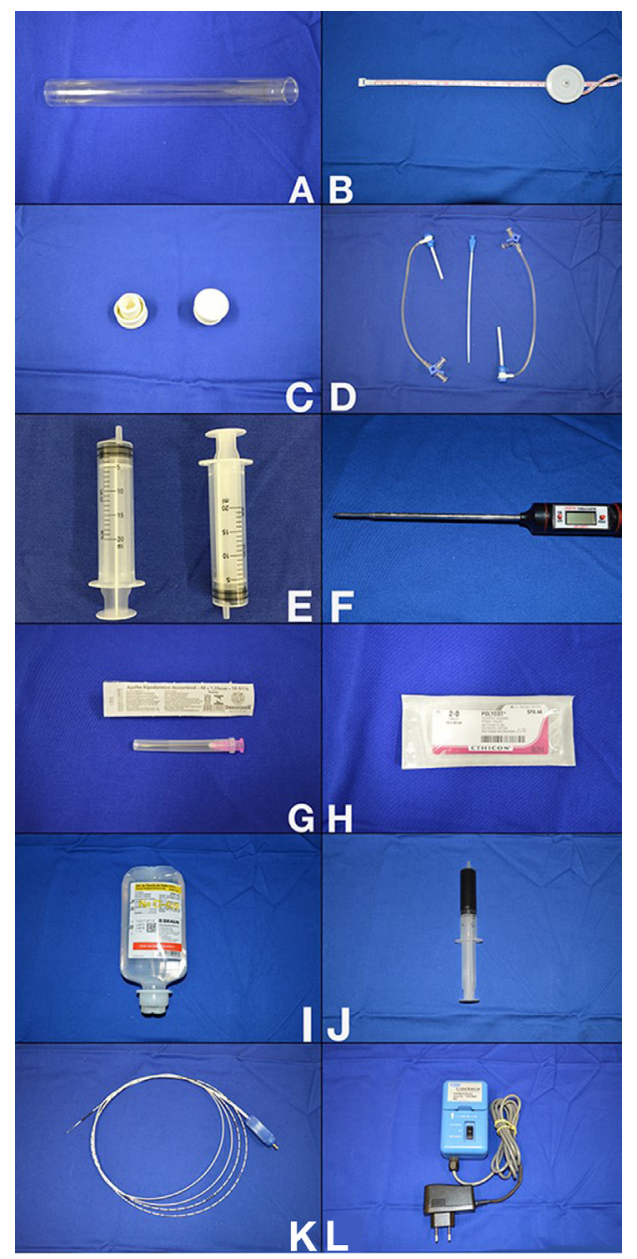

FIGURE 1 - Materials used to produce the experimental model. A) Glass tube $(20 \mathrm{~mm} \times 20 \mathrm{~cm})$ open at both ends; B) Tape measure; C) Two rubber stoppers for glass tube; D) Two short $8 \mathrm{~F}$ introducer sheaths with hemostatic valve; E) Two disposable syringes $(20 \mathrm{~mL})$; F) Digital probe thermometer (temperature range: $-45^{\circ} \mathrm{C}$ to $230^{\circ} \mathrm{C}$ ); G) Disposable hypodermic needle $(1.2 \mathrm{~mm} \times 40 \mathrm{~mm}) ; \mathbf{H}) 2-0$ cotton sutures (no needle); I) $0.9 \%$ saline (100$\mathrm{mL}$ bottle); J) Whole blood collected from a volunteer human donor into a tube containing EDTA as anticoagulant $(20 \mathrm{~mL})$; K) Laser fiber and/ or radiofrequency ablation catheter; L) Automatic pull-back device, for studies involving treatment with endovenous laser.

To prepare the experimental model, initially we cut open a glass tube at both ends (Figure 2A) and insert the $8 \mathrm{~F}$ introducers with hemostatic valve through the rubber stoppers (Figure 2B). The vein to be studied is then passed through the glass tube and stretched, and its ends are fixed in the introducer sheaths with 2-0 cotton sutures (Figure 2C). Then, the rubber stoppers are placed 
at both ends of the tube (Figure 2D) and $0.9 \%$ saline solution is infused into the system through the infusion port of one of the introducers (Figure 2E), followed by puncture of one of the rubber stoppers with a hypodermic needle and infusion of saline into the space around the vein (tumescence) (Figure 2F). A digital probe thermometer is also inserted through one of the rubber stoppers (Figure $2 \mathrm{G}$ ). The laser fiber or radiofrequency ablation catheter is introduced into the vein through one of the introducers (Figure $2 \mathrm{H}$ ) and blood is then injected through the infusion port of the introducer and content is aspirated through the other infusion port to simulate continuous blood flow (Figure 2I). At this point, physiological conditions are established and EVLA can be initiated (Figure 2J).

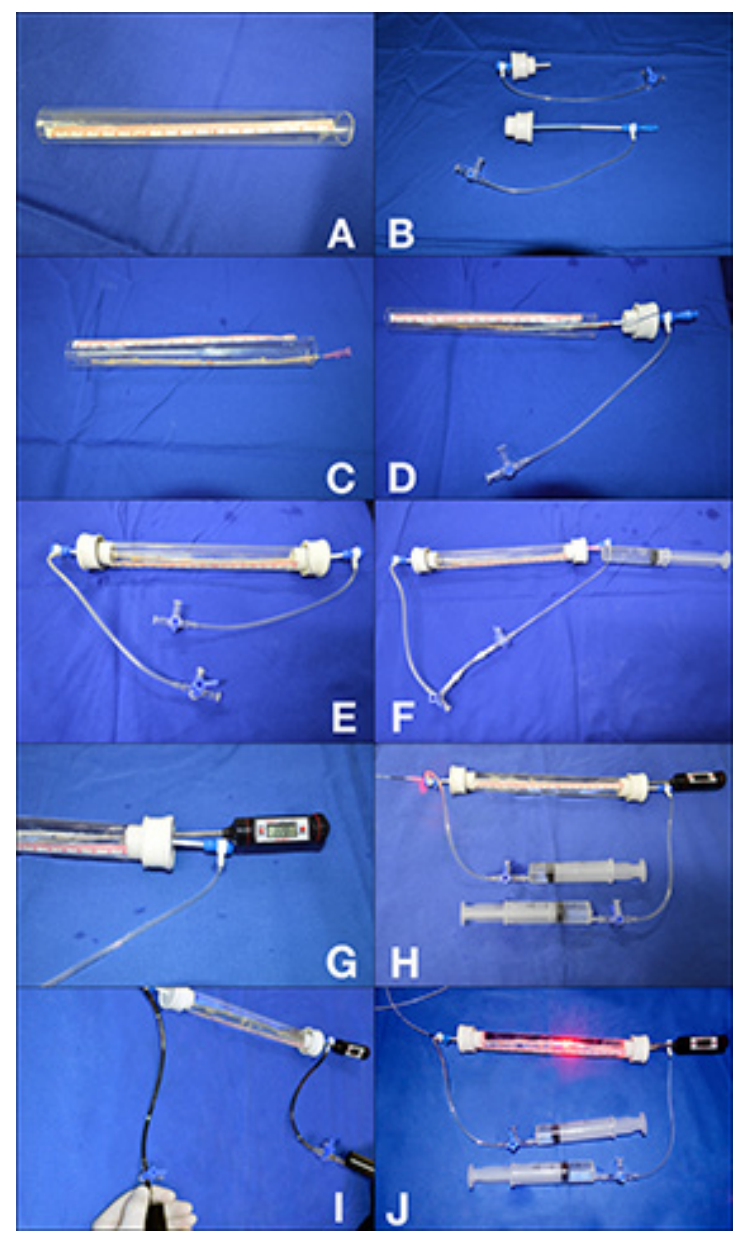

FIGURE 2 - Steps to prepare the experimental model. A) Glass tube cut open at both ends; B) $8 \mathrm{~F}$ introducers with hemostatic valve inserted into the rubber stoppers; C) Vein is passed through the glass tube, stretched, and fixed at both ends in the introducer sheaths; D) Rubber stoppers placed at both ends of the tube; E) Infusion of $0.9 \%$ saline into the system; F) Puncture of one of the rubber stoppers and infusion of saline into the space around the vein (tumescence); G) Digital probe thermometer inserted into one of the rubber stoppers; H) Laser fiber or radiofrequency ablation catheter is passed through one of the introducers; I) Blood is injected through the infusion port of the introducer and content is aspirated through the other infusion port to simulate continuous blood flow; J) Final ex vivo endovenous laser ablation model.

\section{Pilot study}

To assess the reproducibility of the experimental model, a pilot study was conducted to evaluate an ex vivo procedure of EVLA of the GSV with 1470-nm radial fiber laser. A patient with an indication for surgical treatment of varicose veins with stripping of the GSV voluntarily provided written informed consent to and authorization for collection of $20 \mathrm{~mL}$ of blood and use of the stripped GSV in this ex vivo experiment.

During conventional varicose vein surgery, the incompetent GSV was removed, everted, and replaced in its physiological position, being then prepared for later use in the experimental model.

Experimental EVLA was performed using a radial fiber diode laser (Thera Lase, DMC Equipamentos, São CarlosSP, Brazil) set at a wavelength of $1470 \mathrm{~nm}$ to supply $7 \mathrm{~W}$ power and attached to an automatic pull-back device for energy delivery at a speed of $1 \mathrm{~mm} / \mathrm{sec}$. The GSV segment subjected to EVLA was then removed from the experimental model and the central part (approximately $5 \mathrm{~cm}$ of the vein) was cut out and opened longitudinally for macroscopic examination of possible perforations. This section was fixed in $10 \%$ formalin and sent to the laboratory for further histological and immunohistochemical analyses.

At the laboratory, the section was dehydrated in alcohol and embedded in paraffin. Slices were made and stained using the following techniques: hematoxylin \& eosin (H\&E) staining for analysis of tissue morphology, elastic fiber histochemistry for quantification of these proteins, Gomori's trichrome staining for quantification of muscle fibers, and alpha-smooth muscle actin immunohistochemistry for quantification of muscle fibers and expression of the specific marker for this cell type. These techniques allow better visualization of smooth muscle fibers and elastic fibers that make up the wall of the ablated vein.

Slides were evaluated in triplicate, using the color deconvolution plugin for Image $\mathbf{J}$ software to separate colors in photomicrographs. A quantitative analysis was performed on 10 fields in each slide.

\section{Results}

No perforations were observed macroscopically in the GSV segment subjected to EVLA in the pilot study. Confirming this macroscopic finding, no perforations were observed in the microscopic evaluation of this segment by several histological and immunohistochemical methods. 
H\&E staining revealed no smooth muscle cell adhesion, showing tissue disruption (Figure 3).

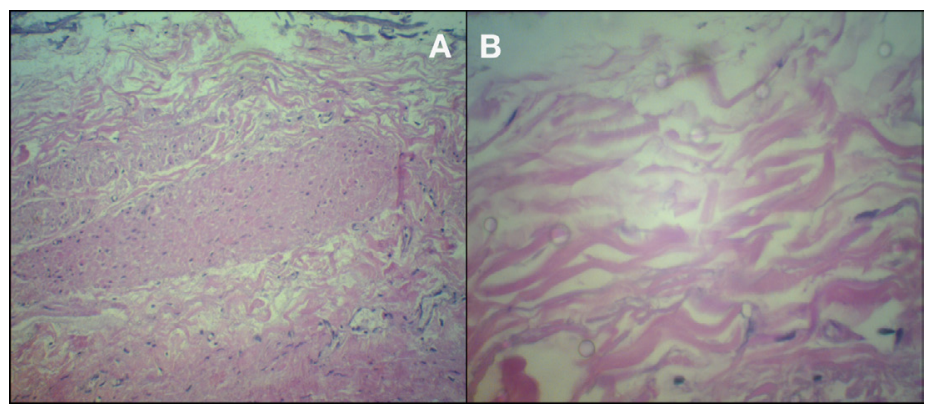

FIGURE 3 - Histological appearance of the central part of the saphenous vein subjected to endovenous laser ablation (hematoxylin \& eosin staining): A) magnification $\times 200$ and B) magnification $\times 400$. Note that there is no smooth muscle cell adhesion, showing tissue disruption.

The histochemical analysis of elastic fibers, in which elastic fibers are light brown in color, showed very low labeling for this protein (Figure 4).

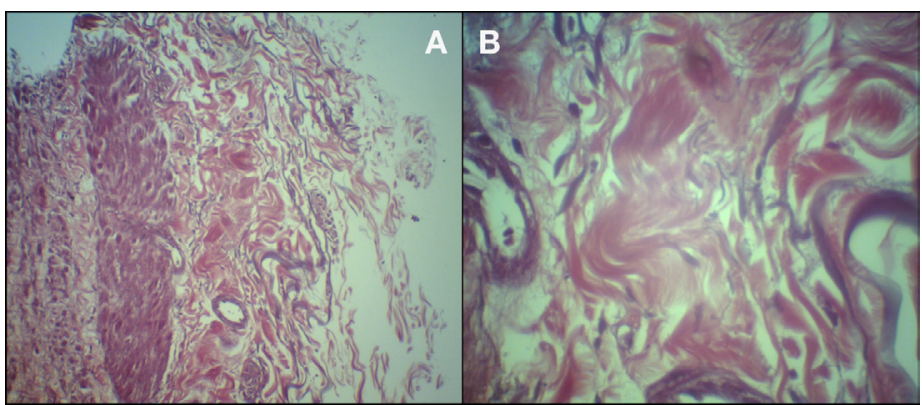

FIGURE 4 - Histochemical analysis of elastic fibers in the central part of the saphenous vein subjected to endovenous laser ablation: A) magnification $\times 200$ and B) magnification $\times 400$. Note the low labeling for elastic fibers (light brown in color).

In Gomori's trichrome staining, the positive reaction for muscle fibers is stained in brown or red. In the analysis of the central part of the ablated GSV, only a small amount of red staining (muscle fibers) was observed (Figure 5).

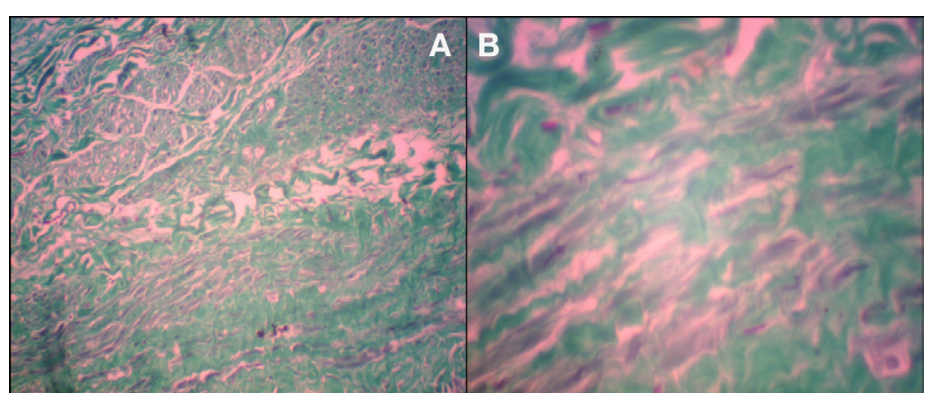

FIGURE 5 - Gomori's trichrome staining in the central part of the saphenous vein subjected to endovenous laser ablation: A) magnification $\times 200$ and B) magnification $\times 400$. Note the small amount of red staining (muscle fibers).
In the immunohistochemical expression of alphasmooth muscle actin, when horseradish peroxidase (HRP) and diaminobenzidine (DAB) are used as markers, brown precipitates are formed where there is interaction with the specific antibody. In the pilot study, disruption of muscle fibers associated with a reduced expression of the specific marker for this cell type was observed in the central part of the GSV segment subjected to EVLA (Figure 6).

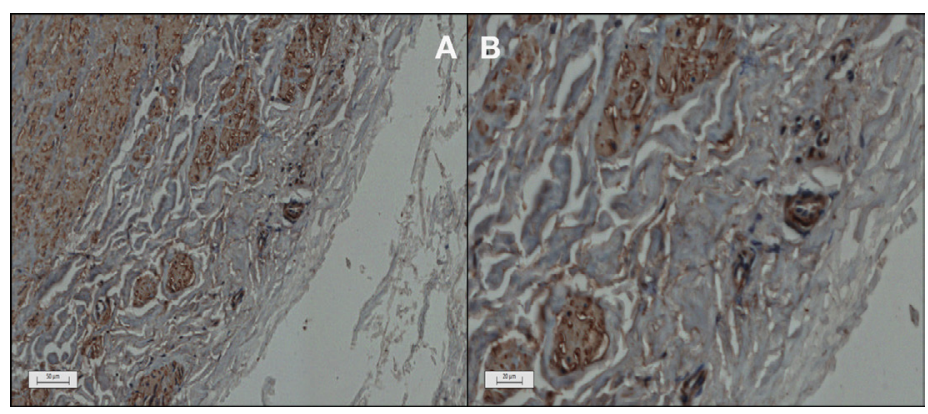

FIGURE 6 - Immunohistochemical expression of alpha-smooth muscle actin in the central part of the saphenous vein subjected to endovenous laser ablation: A) magnification $\times 100$ and B) magnification $\times 200$. Note the disruption of muscle fibers and a reduced expression of the specific marker for this cell type.

\section{Discussion}

The present study investigated an experimental model that allowed the ex vivo performance of EVLA of the GSV and subsequent analysis of macroscopic, histological and immunohistochemical changes in the ablated GSV segment.

New EVLA techniques allow adjustment and combination of multiple parameters that need to be tested and investigated systematically. These techniques should first be tested experimentally under reproducible conditions, especially taking into account macroscopic and histological changes in tissues undergoing EVLA.

Some EVLA parameters are known to affect the outcomes of patients treated with this technique. These parameters include power, wavelength, linear endovenous energy density (LEED), and type of laser fiber ${ }^{3}$.

To reduce possible side effects of EVLA (such as pain, paresthesia, and bruising), devices operating at higher wavelengths and using different types of laser fiber have been developed. Theoretically, higher wavelengths target the interstitial water in the vessel wall as the chromophore, reducing side effects ${ }^{4}$. Because the use of a conventional bare-tip fiber often leads to 
uneven application of energy in the vein wall, resulting in local perforation and uneven destruction of the perivenous tissues, new fibers have been developed in order to reduce these effects ${ }^{5-7}$.

EVLA at low energy densities results in lower occlusion rates and higher recurrence rates than higher energy $\mathrm{EVLA}^{8,9}$. Park et al. ${ }^{10}$ concluded that EVLA with the use of 1470-nm laser and low energy (LEED of $80 \mathrm{~J} / \mathrm{cm}$ or lower) is an effective, safe, and technically successful option for the treatment of incompetent saphenous veins.

In order to scientifically investigate different EVLA parameters, several experimental models have been described. Rossi et $a l .{ }^{11}$ investigated the effects of an experimental electrocautery developed to cause endovascular venous thermal injury in a bovine saphenous vein model. Macroscopic and microscopic effects were analyzed. The higher the electric energy applied through the endovascular electrocautery, the greater the nuclear pyknosis and more intense the cytoplasmic shrinkage. The authors concluded that this method was able to induce the destruction of the intima of bovine saphenous vein and provoke endovascular thermal injury.

Sroka et al. ${ }^{12}$ developed a model using ox foot veins to investigate endoluminal treatment of varicose veins. Ox feet are byproducts in slaughterhouses and are available in large numbers and at low cost. The model had some limitations, such as limited time to perform the procedure after the animal's death and inability to study in vivo the reaction of the organism to the procedure or long-term effects of the treatment. Also, despite the anatomical and functional analogy, there are differences between humans and oxen. Therefore, the results of the experiment cannot be extrapolated to the clinical setting without restriction.

An alternative to experiments in animal organ-models is the use of human tissue explants harvested during surgery ${ }^{13}$. Massaki et al. ${ }^{14}$ used an ex vivo model with human veins harvested during outpatient phlebectomy procedures. The veins were sutured at one end, suspended by a weighting device and placed in a column of warm saline. The lumen of each vein was then filled with porcine blood and a fiber attached to an automatic pullback device was inserted into the lumen. Similar volume of blood was injected into each vein segment using a hypodermic syringe in order to simulate residual blood that could act as an absorbing chromophore.

Yamamoto and Sakata investigated the influence of fibers and wavelengths on the mechanism of action of EVLA using a model consisting of acrylic tubes, rubber stoppers, injection needles, and 7F sheaths. The model was able to reproduce the physiological conditions of EVLA of the GSV, except for the fact that the system was static without blood flow. Segments of the GSV were passed through the tube, and the vein was then stretched and fixed at both sides of the sheath. After filling the tube with saline at room temperature, human blood with EDTA as anticoagulant from a volunteer donor was injected through the 7F sheath. The sheaths were left open during the experiment to prevent steam condensation ${ }^{15}$.

The experimental model described in the present study shows some similarities with the model described by Yamamoto and Sakata ${ }^{15}$. However, some innovations were introduced, and the main changes proposed are as follows:

1) Use of a larger glass tube so that a longer vein segment can be tested, allowing the application of different treatment parameters to the same vein segment during the study. We also aimed to expand the experimental set examined in the study by using other research resources, such as a digital thermometer inside the tumescent fluid, providing the possibility of studying different types of tumescent fluid as well as its temperature variation before, during and after EVLA;

2) Use of large-diameter $(8 \mathrm{~F})$ introducer sheaths with hemostatic valve, thus allowing and facilitating continuous infusion of blood into the system;

3) Injecting blood through one of the infusion ports of the introducer and aspirating the content through the other infusion port to simulate continuous and dynamic blood flow, which remains active at least at the start of EVLA, mimicking the physiological conditions of the procedure;

4) Possibility of using two digital thermometers to measure the temperature both of the tumescent fluid, by introducing the thermometer through the rubber stopper, and of blood in the system, by introducing the thermometer through the sheath, thus allowing the measurement of blood temperature in the system at the beginning and end of EVLA;

5) Possibility of making future adjustments to investigate acute ex vivo changes in veins subjected to other modern methods of endovenous treatment, such as radiofrequency ablation ${ }^{16}$, polidocanol foam sclerotherapy ${ }^{17}$, and the ClariVein ${ }^{\circledR}$ catheter an endoluminal mechanico-chemical obliteration technique ${ }^{18}$, especially considering the maintenance of blood flow simulating physiological conditions.

Carvalho et al.$^{18}$ studied morphological and histochemical changes in normal and varicose veins and concluded that, in 
varicose veins, there was a relative increase in smooth muscle in thickened segments and muscle fibers were more strongly stained than those of normal veins, as shown by Gomori's trichrome staining. Additionally, elastic fibers, which were normally present in the media and adventitia of the normal saphenous vein, exhibited a fragmented pattern in varicose veins ${ }^{18}$.

This pilot study found that, after laser thermal ablation, no muscle cell adhesion was observed, showing tissue disruption in hematoxylin \& eosin staining. There were also a decrease in the number of muscle fibers, a small amount of red staining according to Gomori's trichrome staining, low labeling for elastic cells according to elastic fiber histochemistry, and reduced expression of alpha smooth muscle actin. These changes in GSV wall may progress to thickening and fibrosis, leading to non-thrombotic occlusion.

\section{Limitations}

Although ex vivo experiments look promising, they have some limitations, including: the vein segment may undergo some changes during harvesting; analysis of only immediate effects of the treatment; and inability to study the perivascular tissue.

\section{Conclusions}

The ex vivo endovenous laser ablation model described here is suitable for use in scientific research and for medical training purposes considering the use of different EVLA parameters. In addition, it may be used for preclinical testing based on acute macroscopic, histological and immunohistochemical findings. It is therefore a low cost alternative to in vivo experiments, providing standardized experimental conditions.

\section{References}

1. Evans CJ, Fowkes FG, Ruckley CV, Lee AJ. Prevalence of varicose veins and chronic venous insufficiency in men and women in the general population: Edinburgh Vein Study. J Epidemiol Community Health. 1999 Mar;53(3):149-53. PMID: 10396491.

2. van den Bos R, Arends L, Kockaert M, Neumann M, Nijsten T. Endovenous therapies of lower extremity varicosities: a metaanalysis. J Vasc Surg. 2009 Jan;49(1):230-9. doi: 10.1016/j. jvs.2008.06.030.

3. Kabnick LS. Which is more important for postoperative recovery: laser Wavelength or Fibers? J Vas Surg. 2012 Jan;55(1):307. doi: http://dx.doi.org/10.1016/j.jvs.2011.11.040.

4. Vuylsteke ME, Mordon SR. Endovenous laser ablation: a review of mechanisms of action. Ann Vasc Surg. 2012 Apr;26(3):424-33. doi: 10.1016/j.avsg.2011.05.037.
5. Vuylsteke ME, Thomis S, Mahieu P, Mordon S, Fourneau I. Endovenous laser ablation of the great saphenous vein using a bare fibre versus a tulip fibre: a randomised clinical trial. Eur J Vasc Endovasc Surg. 2012 Dec;44(6):587-92. doi: 10.1016/j. ejvs.2012.09.003.

6. Goldman MP, Mauricio M, Rao J. Intravascular 1320-nm laser closure of the great saphenous vein: a 6- to 12-month follow-up study. Dermatol Surg. 2004 Nov;30(11):1380-5. PMID: 15522018.

7. Pannier F, Rabe E, Maurins U. First results with a new 1470-nm diode laser for endovenous ablation of incompetent saphenous veins. Phlebology. 2009 Feb;24(1):26-30. doi: 10.1258/ phleb.2008.008038.

8. Proebstle TM, Moehler T, Gül D, Herdemann S. Endovenous treatment of the great saphenous vein using a $1,320 \mathrm{~nm} \mathrm{Nd:YAG}$ laser causes fewer side effects than using a $940 \mathrm{~nm}$ diode laser. Dermatol Surg. 2005 Dec;31(12):1678-83; discussion 1683-4. PMID: 16336887.

9. Timperman PE. Prospective evaluation of higher energy great saphenous vein endovenous laser treatment. J Vasc Interv Radiol. 2005 Jun;16(6):791-4. PMID: 15947042.

10. Park JA, Park SW, Chang IS, Hwang JJ, Lee SA, Kim JS, Chee HK, Yun IJ. The 1,470-nm bare-fiber diode laser ablation of the great saphenous vein and small saphenous vein at 1-year follow-up using 8-12 W and a mean linear endovenous energy density of $72 \mathrm{~J} / \mathrm{cm}$. J Vasc Interv Radiol. 2014 Nov;25(11):1795-800. doi: 10.1016/j. jvir.2014.07.009.

11. Rossi FH, Izukawa NM, Silva DG, Chen J, Prakasan AK, Zamorano MM, Silva LM. Effects of electrocautery to provoke endovascular thermal injury. Acta Cir Bras. 2011 Spt/Oct;26(5):329-32. doi: 10.1590/S0102-86502011000500001.

12. Sroka R, Weick K, Steckmaier S, Steckmaier B, Blagova R, Sroka I, Sadeghi-Azandaryani M, Maier J, Schmedt CG. The ox-footmodel for investigating endoluminal thermal treatment modalities of varicosis vein diseases. ALTEX. 2012;29(4):403-10. PMID: 23138510 .

13. Proebstle TM, Lehr HA, Kargl A, Espinola-Klein C, Rother W, Bethge S, Knop J. Endovenous treatment of the greater saphenous vein with a 940-nm diode laser: thrombotic occlusion after endoluminal thermal damage by laser-generated steam bubbles. J Vasc Surg. 2002 Apr;35(4):729-36. PMID: 11932671.

14. Massaki AB, Kiripolsky MG, Detwiler SP, Goldman MP. Endoluminal laser delivery mode and wavelength effects on varicose veins in an ex vivo model. Lasers Surg Med. 2013 Feb;45(2):123-9. doi: 10.1002/lsm.22069.

15. Yamamoto T, Sakata M. Influence of fibers and wavelengths on the mechanism of action of endovenous laser ablation. J Vasc Surg. 2014 Jan;2(1):61-9. doi: http://dx.doi.org/10.1016/j.jvsv.2013.05.009.

16. Sroka R, Schmedt CG, Steckmeier S, Meissner OA, Beyer W, Babaryka G, Steckmeier B. Ex-vivo investigation of endoluminal vein treatment by means of radiofrequency and laser irradiation. Med Las Appl. 2006 April;21(1):15-22. doi: doi:10.1016/j. mla.2005.11.003.

17. Erkin A, Kosemehmetoglu K, Diler MS, Koksal C. Evaluation of the minimum effective concentration of foam sclerosant in an exvivo study. Eur J Vasc Endovasc Surg. 2012 Dec;44(6):593-7. doi: 10.1016/j.ejvs.2012.09.021

18. Kendler M, Averbeck M, Simon JC, Ziemer M. Histology of saphenous veins after treatment with the ClariVein ${ }^{\circledR}$ device - an exvivo experiment. J Dtsch Dermatol Ges. 2013 Apr;11(4):348-52. doi: $10.1111 /$ ddg. 12022 . 
19. Carvalho JJ, Rodrigues MI, Cotta Pereira G, Magalhães LO, Panico MD, Silveira PR, Medeiros A. Estudo histoquímico das fibras do sistema elástico e da proteoglicanas da parede de veias safenadas normais e alteradas. Cir Vasc Ang. 1988;4(2):7-12.

\section{Correspondence:}

Walter Junior Boim Araujo

Rua das Aroeiras, 531 Quadra 55, Lote 17

83327-115 Pinhais - PR Brasil

Tel.: (55 41)3244-5000

wboim@hotmail.com

Received: Nov 08, 2015

Review: Jan 10, 2016

Accepted: Feb 11, 2016

Conflict of interest: none

Financial source: CAPES

${ }^{1}$ Research performed at Department of Surgery, Universidade Federal do Paraná (UFPR), Curitiba-PR, Brazil. Part of PhD degree thesis, Postgraduate Program in Clinical Surgery. Tutor: Jorge Rufino Ribas Timi. 